\title{
Local Chain Dynamics of Poly $(N$-vinylcarbazole) Studied by the Fluorescence Depolarization Method
}

\author{
Hiroyuki AoKI, Jun-ichi HoRINAKA, Shinzaburo Ito ${ }^{\dagger}$ Masahide YAMAMOTo, ${ }^{*}$ \\ Hiroshi Katayama, Masami Kamigaito, and Mitsuo SaWamoto \\ Department of Polymer Chemistry, Graduate School of Engineering, \\ Kyoto University, Sakyo-ku, Kyoto 606-8501, Japan \\ * Faculty of Science and Engineering, Ritsumeikan University, Kusatsu, \\ Shiga 520-8577, Japan
}

(Received November 13, 2000; Accepted April 2, 2001)

\begin{abstract}
The local segmental motion at the center of the main chain of poly $(N$-vinylcarbazole) (PVCz) in dilute solutions was examined by the time-resolved fluorescence depolarization method. Anthracene-labeled PVCzs with various molecular weights were synthesized by living cationic polymerization. The relaxation time and the activation energy of the local motion were evaluated through the fluorescence anisotropy decay. The molecular weight dependence of the relaxation time could not be explained merely from the viewpoint of segment density, suggesting that the local potential for the rotational motion is an important factor governing the local dynamics of the polymer chain. The local motion of $\mathrm{PVCz}$ was compared with those of other polymers. Both the relaxation time and the activation energy were larger than those of other polymers. Moreover, PVCz had a larger critical molecular weight which is defined as the molecular weight where the reduced relaxation time reaches the saturation value. This is attributed to the fact that the bulky side chain suppresses the conformational transition of the polymer backbone.

KEY WORDS Fluorescence Depolarization / Poly $(N$-vinylcarbazole $)$ / Local Motion / Molecular Weight Effect / Dilute Solution / Anthracence Probe /
\end{abstract}

Poly $(N$-vinylcarbazole) (PVCz) has been gathering much attention as a photofunctional polymer, ${ }^{1,2}$ and many fundamental, ${ }^{3-6}$ and practical $^{7-9}$ studies have been performed. For understanding the basis of photofunctional processes, the dynamic properties as well as the static properties of the polymer chain have to be fully understood. Generally, the dynamics of polymers have been extensively studied from the theoretical ${ }^{10-12}$ and experimental points of view. The local chain dynamics of flexible polymers has been experimentally studied by various techniques such as $\mathrm{NMR},{ }^{13-15} \mathrm{ESR},{ }^{16,17} \mathrm{di}-$ electric relaxation, ${ }^{18}$ neutron scattering, ${ }^{19}$ light scattering, ${ }^{20,21}$ and fluorescence method. ${ }^{2,22-30}$ The timeresolved fluorescence depolarization method enables one to measure directly the orientational autocorrelation function of the fluorescent probe introduced into the polymer in a time range of $10^{-10}-10^{-7} \mathrm{~s}$. We have examined the local dynamic behavior of the polymer chain by this method using a fluorescent probe introduced into the polymer chains of polystyrene (PS), poly(cis-1,4isoprene) (PI), poly(oxyethylene) (POE), and poly(methyl methacrylate) (PMMA). ${ }^{23-28} \mathrm{PVCz}$ has bulky carbazolyl groups as the side chains. The static property of $\mathrm{PVCz}$ in dilute solutions ${ }^{1}$ was investigated by several workers, however, few studies on the dynamic behavior of $\mathrm{PVCz}$ have been reported. ${ }^{31}$ It is interesting to know the extent to which the rotational relaxation of the polymer backbone is affected by the substituent.

In the present study, the local chain dynamics of $\mathrm{PVCz}$ in dilute solutions was examined by the fluorescence depolarization method. The sample polymer was labeled at the middle of the chain with an anthracene probe by the coupling reaction of living ends of $\mathrm{PVCz}$. The transition moment of the introduced probe lies along the main chain. Therefore, the obtained fluorescence anisotropy represents the orientational autocorrelation function for the middle segment of the main chain. The molecular weight dependence of the local motion of $\mathrm{PVCz}$ was examined in the $\Theta$ and good solvents, and the factors that governs the local chain dynamics were discussed. The characteristics of the dynamics of $\mathrm{PVCz}$ were also compared with those of other polymers.

\section{EXPERIMENTAL}

\section{Materials}

Figure 1 shows the chemical structure of $\mathrm{PVCz}$ labeled with anthracene at the center of the chain. The doubleheaded arrow in Figure 1 indicates the transition moment for the anthracene group. The polymers were prepared by living cationic polymerization of $\mathrm{N}$ vinylcarbazole initiated by $\mathrm{HI}$ in $\mathrm{CH}_{2} \mathrm{Cl}_{2}$ at $-48{ }^{\circ} \mathrm{C} .{ }^{32} \mathrm{~N}$ Vinylcarbazole (Nacalai Tesque) was recrystallized four times from hexane, and $\mathrm{CH}_{2} \mathrm{Cl}_{2}$ was doubly distilled before use. Anthracene was incorporated into the middle of the main chain by the coupling the living ends of $\mathrm{PVCz}$ with 9,10-anthracenedithiol lithium salt, which was syn-

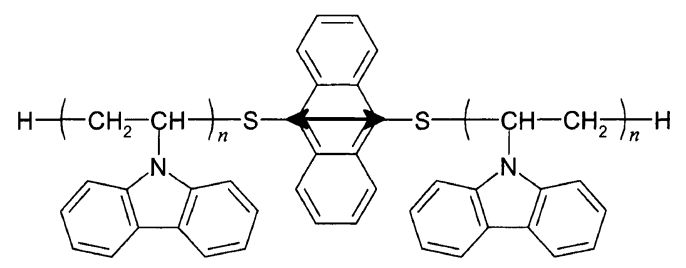

Figure 1. Molecular structure of PVCz labeled with anthracene at the center of the main chain.

\footnotetext{
${ }^{\dagger}$ To whom correspondence should be addressed (Phone: +81-75-753-5612, Fax: +81-75-753-5632, E-mail: sito@polym.kyoto-u.ac.jp).
} 
Table I. Characterization of The Samples

\begin{tabular}{lcc}
\hline & $M_{\mathrm{w}} \times 10^{-3}$ & $M_{\mathrm{w}} / M_{\mathrm{n}}$ \\
\hline PVCz-09 & 9.2 & 1.12 \\
PVCz-22 & 21.7 & 1.03 \\
PVCz-42 & 41.6 & 1.06 \\
PVCz-103 & 103 & 1.03 \\
\hline
\end{tabular}

thesized by addition of $n$-butyl lithium to 9,10 anthracenedithiol. ${ }^{33}$ The GPC curve for the obtained polymer had two peaks. The peak at the lower molecular weight side was attributed to the $\mathrm{PVCz}$ chain unlabeled or labeled at the one end. Only a higher molecular weight side peak, which was attributed to $\mathrm{PVCz}$ labeled at the chain center, was fractionated by GPC. The molecular weight was estimated from GPC measurement calibrated with the narrow molecular weight distributed $\mathrm{PVCz}$ standards whose molecular weights were determined by light scattering experiments. The fraction of racemic diads was estimated to be 0.63 independent of molecular weight from ${ }^{1} \mathrm{H}$ NMR measurement. ${ }^{34}$ Table I summarizes the weight-averaged molecular weights and the molecular weight distributions for the samples used in this study.

\section{Fluorescence Depolarization Measurement}

Toluene (Dojin, spectrophotomeric grade) and THF (Wako, guaranteed grade) were used as the solvents. Toluene was used without further purification and THF was twice distilled before use. The concentration of the sample solution was $c a \cdot 10^{-5} \mathrm{M}$, so that the fluorescence depolarization by the energy migration among anthracene probes $^{35}$ could be avoided. The solution was put into a quartz cell and degassed. The fluorescence anisotropy decay was measured by the time-correlated single photon counting technique. ${ }^{36}$ A frequency doubled Ti: sapphire laser (Tsunami, Spectra Physics Inc.) was used as a light source at a wavelength of $410 \mathrm{~nm}$. The anthracene fluorescence from the sample was detected by a microchannel plate photomultiplier tube (R3809, Hamamatsu Photonics K. K.). The fluorescence signal and the excitation pulse signal detected by a photodiode (AR-S2, Antel Optronics Inc.) were inputted to a time-toamplitude converter (Model 457, Ortec) as the start and stop signals, respectively. The FWHM of the instrumental function was ca. $60 \mathrm{ps}$. The fluorescence components parallel and perpendicular to the vertically polarized excitation pulse were measured alternately to avoid the data distortion due to the time drift. The measurement temperature was controlled within $\pm 0.1^{\circ} \mathrm{C}$ by a water circulating system.

\section{Data Analysis}

The fluorescence anisotropy, $r(t)$, is defined as

$$
r(t)=\frac{I_{\|}(t)-I_{\perp}(t)}{I_{\|}(t)+2 I_{\perp}(t)}
$$

where $I_{\|}(t)$ and $I_{\perp}(t)$ are the parallel and perpendicular component of the fluorescence intensity to the excitation polarization direction, respectively. The relaxational process of the polymer chain consists of various motional

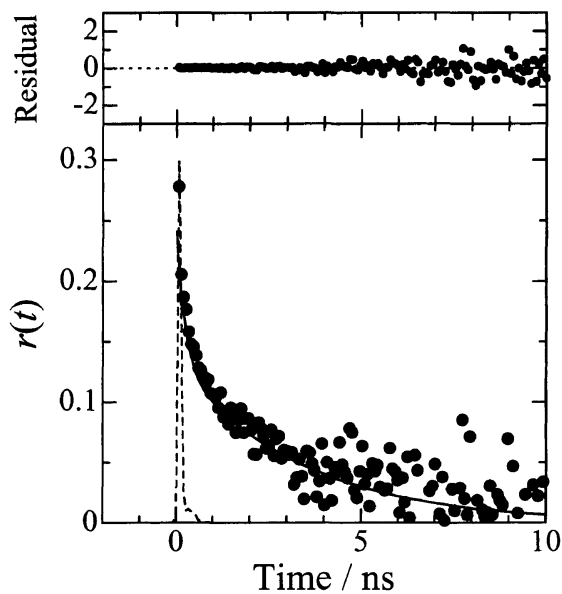

Figure 2. A fitting example of $r(t)$ with two-component exponential functions. The filled circles are the observed $r(t)$ for PVCz-42 in $\mathrm{THF}$ at $17.4^{\circ} \mathrm{C}$. The broken and solid lines indicate the instrumental function and the fitting curve, respectively. The fitting parameters are $r_{0}=0.241, x=0.554, T_{1}=3.34 \mathrm{~ns}$, and $T_{2}=0.225 \mathrm{~ns}$.

modes, so $r(t)$ may consist of the sum of two or more exponential functions. However, practically, the experimental data fitted well to a double exponential function,

$$
r(t)=r_{0}\left[x \exp \left(-\frac{t}{T_{1}}\right)+(1-x) \exp \left(-\frac{t}{T_{2}}\right)\right]
$$

where $T_{1}>T_{2}$. Figure 2 shows a fitting example of $r(t)$ for $\mathrm{PVCz}-42$ in $\mathrm{THF}$ solvent at $17.4^{\circ} \mathrm{C}$, where the longer and shorter component of the relaxation time, $T_{1}$ and $T_{2}$, were $c a .3 \mathrm{~ns}$ and $c a .0 .2 \mathrm{~ns}$, respectively, with the fraction of the longer component $x=0.55$. The ratio of $T_{1}$ to $T_{2}$ and the fraction $x$ were nearly independent of temperature, but dependent on the solvent. The detailed behavior of $T_{1}, T_{2}$, and $x$ is being studied and will be reported later. In this study, the mobility of the polymer chain was discussed in terms of the mean relaxation time, $T_{\mathrm{m}}$, defined as eq 3 and calculated by eq 4 .

$$
\begin{aligned}
T_{\mathrm{m}} & =r_{0}{ }^{-1} \int_{0}^{\infty} r(t) \mathrm{d} t \\
& =x T_{1}+(1-x) T_{2}
\end{aligned}
$$

\section{RESULTS AND DISCUSSION}

\section{Reduced Relaxation Time}

The previous studies showed that the relaxation time for the local motion of polymers in a low viscosity solvent, $<1 \mathrm{cP}\left(\mathrm{cP}=10^{-3} \mathrm{~Pa} \mathrm{~s}\right)$, is proportional to the solvent viscosity, $\eta .^{24,29,37}$ Hence, the local dynamics of a polymer chain can be discussed in terms of the reduced relaxation time, $T_{\mathrm{m}} / \eta$. Figure 3 shows the molecular weight dependence of $T_{\mathrm{m}} / \eta$ for the toluene and THF solutions at $37^{\circ} \mathrm{C}$. In this plot, the reduced relaxation time for 9,10-dimethylanthracene is also indicated as a diamond symbol. The value of $T_{\mathrm{m}} / \eta$ for 9,10 dimethylanthracene, which provides the limiting value 


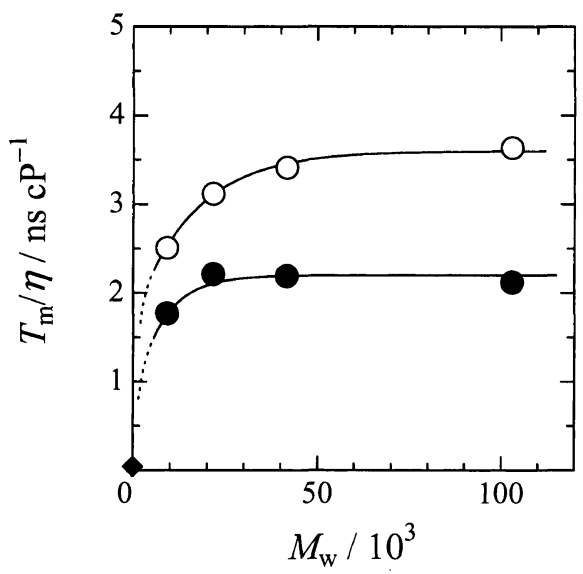

Figure 3. Molecular weight dependence of the reduced relaxation time, $T_{\mathrm{m}} / \eta$, at $37^{\circ} \mathrm{C}$. The open and filled circles indicate the data for toluene and THF solution, respectively. A diamond symbol $\checkmark$ indicates the reduced relaxation time for 9,10dimethylanthracene estimated from a steady state fluorescence depolarization measurement.

for low molecular weight PVCz, was estimated to be $c a$. $0.04 \mathrm{~ns} \mathrm{cP}^{-1}$ from the steady state fluorescence anisotropy measurement. Toluene is a $\Theta$ solvent at $37^{\circ} \mathrm{C}$, and THF is a good solvent. ${ }^{38,39} \mathrm{In} \mathrm{THF}$, the value of $T_{\mathrm{m}} / \eta$ increased with the increase of molecular weight in the low molecular weight region. In this region, it seems that both the local backbone motion and the entire rotational motion of the polymer chain contribute to the mean relaxation time. Then, the reduced relaxation time asymptotically reached $T_{\mathrm{m}} / \eta=2.3 \mathrm{~ns} \mathrm{cP^{-1 }}$ at $M_{\mathrm{w}}=25000$. The saturation of $T_{\mathrm{m}} / \eta$ in the high molecular weight region indicates that the fluorescence depolarization method measures the local motion of the PVCz backbone which occurs within the scale of the molecular weight of 25000 . The value of $T_{\mathrm{m}} / \eta$ for the toluene solution also increased with the increase of molecular weight in the range of $M_{\mathrm{w}}$ $<c a$. 50000, and gradually reached a constant value, $T_{\mathrm{m}} / \eta=3.6 \mathrm{~ns} \mathrm{cP}^{-1}$. Waldow et al. examined the local motion of PI by the fluorescence depolarization method and showed that the relaxation time increased with the increase of molecular weight in a $\Theta$ solvent while the relaxation time was independent of molecular weight in a good solvent. They explained the molecular weight dependence of the relaxation time for PI in terms of the chain segment density around the fluorescent probe, i.e., the segment density at the center of the unperturbed chain monotonically increased with the increase of molecular weight. ${ }^{40}$ In the case of $\mathrm{PVCz}$, however, $T_{\mathrm{m}} / \eta$ in the $\Theta$ state tended to reach a constant value in the range of $M_{\mathrm{w}}>50000$. This indicates that the segment density is not an unique factor governing the local chain dynamics. The motional modes for the local chain dynamics consist of the conformational transition across a potential barrier and the librational motion within a potential well. ${ }^{12}$ Recent molecular dynamics simulations showed that the libration plays an important role in the local chain motion as well as the conformational transition. ${ }^{41-43} \mathrm{Be}-$ cause the libration is the motion that occurs within a potential well, it is dependent upon the shape of the local potential. The local motion of the polymer chain on a nanosecond scale is influenced by the potential energy, which is affected by the solvent condition as well as by the molecular structure of the polymer.

Now we consider the difference in $T_{\mathrm{m}} / \eta$ for $\mathrm{PVCz}$ in the two solvents in the range of $M_{\mathrm{w}}<20000$. Abe et al. have studied the excluded-volume effect in a wide molecular weight range for PS and PMMA. They examined the solvent dependence of the mean-square radius of gyration, $\left\langle s^{2}\right\rangle$, and the intrinsic viscosity, $[\eta]{ }^{44,45}$ They showed that the values of $\left\langle s^{2}\right\rangle$ or $[\eta]$ were almost independent of the solvent condition in the oligomer region, indicating that the chain dimension even in a good solvent agreed with that of the unperturbed polymer chain for the two polymers. Therefore, it seems reasonable to be said that in the low molecular weight region the dimension and conformation of $\mathrm{PVCz}$ in toluene, $\Theta$ solvent, are similar to those in THF, a good solvent. Therefore, if the local motion were governed only by the segment density, the reduced relaxation times in $\Theta$ and good solvents would be the same in the oligomer region. However, as shown in Figure 3, the reduced relaxation time, $T_{\mathrm{m}} / \eta$, for $\mathrm{PVCz}$ in the low molecular weight region was dependent on the solvent quality. This indicates that the contribution of the segment density is small and that the local motion of $\mathrm{PVCz}$ is affected by the local potential energy. Then, it is probable that the solvent quality affects the potential energy, that is, the poor solvent quality probably causes the high potential energy, resulting in a large $T_{\mathrm{m}} / \eta$ in a $\Theta$ solvent.

It should be noted that the relationships of $T_{\mathrm{m}} / \eta$ against molecular weight for the toluene and THF solutions give a different critical molecular weight, $M_{\mathrm{c}}$, which is defined as the molecular weight where the reduced relaxation time reaches an asymptotic value. The value of $M_{\mathrm{c}}$ in toluene is larger than that in THF, that is, the values of $T_{\mathrm{m}} / \eta$ increased more gradually with the increase of molecular weight in toluene than in THF. The polymer chain in a poor solvent takes a contracted conformation compared to that in a good solvent. In the high molecular weight region, the interaction with the segments apart from the chain center affects the motion at the middle of the main chain. This probably causes the difference in $M_{c}$ between good and $\Theta$ solvents.

\section{Activation Energy}

The activation energy for the local segmental motion was estimated according to Kramers' diffusion limit. ${ }^{11,46}$ The activation energy, $E^{*}$, is related to the relaxation time, $T_{\mathrm{m}}$, by the following equation.

$$
T_{\mathrm{m}} / \eta=A \exp \left(\frac{E^{*}}{R T}\right)
$$

where $A, R$, and $T$ are a constant, the gas constant, and the absolute temperature, respectively. Figure 4 shows the Arrhenius plot of $T_{\mathrm{m}} / \eta$ for PVCz in the THF solvent. The value of $E^{*}$ was evaluated from the slope of this plot. The relationship between $E^{*}$ for the THF solution and the molecular weight is shown in Figure 5. The molecular weight dependence of $E^{*}$ was similar to that of $T_{\mathrm{m}} / \eta$, that is, the value of $E^{*}$ for THF solution was saturated at $M_{\mathrm{w}}=24000$. On the other hand, the activation energy obtained for $\mathrm{PVCz}$ in toluene above the $\Theta$ 


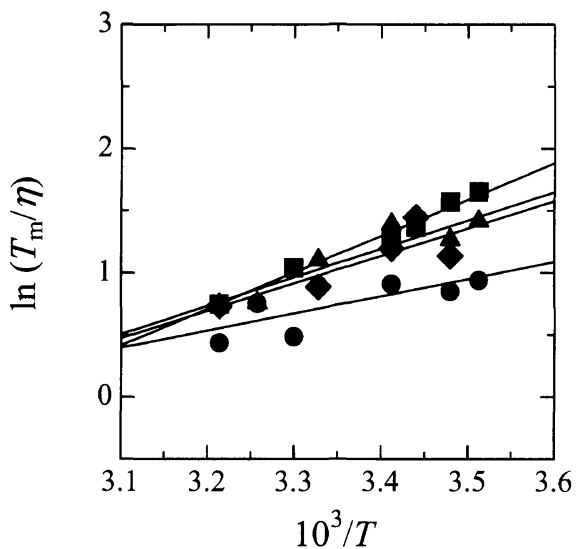

Figure 4. Arrhenius plots of $T_{\mathrm{m}} / \eta$ for PVCz-09(-), $-22(\boldsymbol{\Delta}),-42$ $(\square),-103(\diamond)$ in THF solvent.

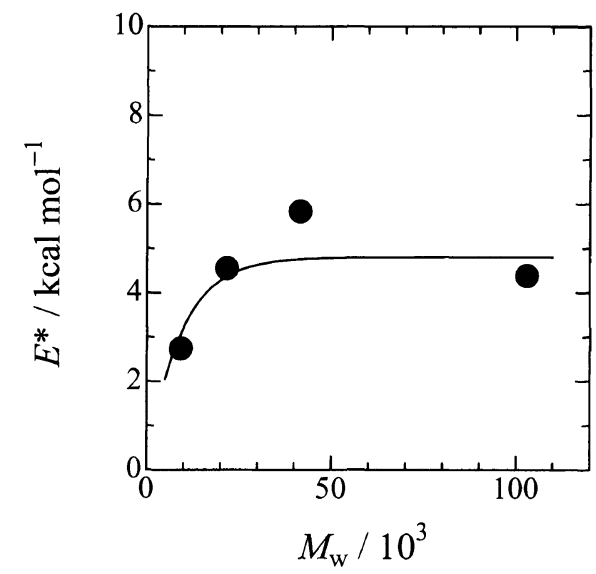

Figure 5. Molecular weight dependence of the activation energy, $E^{*}$, for $\mathrm{PVCz}$ in THF. The value of $E^{*}$ was evaluated from the slope of the Arrhenius plot in Figure 4.

temperature, $37.5^{\circ} \mathrm{C}$, was considerably scattered due to high temperature. The value of $E^{*}$ obtained below the $\Theta$ temperature did not reflect the activation energy for the local segmental motion of $\mathrm{PVCz}$ because of the poor solubility, so the activation energy in toluene is not discussed here.

Karali et al. studied ${ }^{13} \mathrm{C}$ NMR on local chain dynamics of PVCz. ${ }^{31}$ They reported the activation energy of 2.2 $\mathrm{kcal} \mathrm{mol}^{-1}$ for the $\mathrm{C}-\mathrm{H}$ vector orientation dynamics, which was smaller than that evaluated in this study. This was probably attributed to the difference in the probed motional mode. The fluorescence depolarization directly probes the motion of the main chain, while NMR measurement observes the motional modulation of the ${ }^{13} \mathrm{C}-{ }^{1} \mathrm{H}$ dipolar interaction.

\section{Comparison with Other Polymers}

The reduced relaxation time and the activation energy for $\mathrm{PVCz}$ were compared with those for other polymers; $\mathrm{POE},{ }^{23} \mathrm{PI},{ }^{24} \mathrm{PS},{ }^{25,26}$ and $s$-PMMA. ${ }^{27}$ All the polymers were labeled with anthracene group at the center of the main chain, and they had a sufficiently large molecular weight. Table II shows the reduced relaxation time at $20^{\circ} \mathrm{C}$ and the activation energy for each polymer in good solvents evaluated by the fluorescence depolarization method. For comparison of the reduced relaxation time
Table II. Reduced Relaxation Time, $T_{\mathrm{m}} / \eta$, Measured at $20^{\circ} \mathrm{C}$ and Activation Energy, $E^{*}$, for Several Polymers in Good Solvents

\begin{tabular}{llcc}
\multicolumn{3}{c}{ Activation Energy, $E^{2}$, for Several Polymers in Good Solvents } \\
\hline POE & Solvent & $T_{\mathrm{m}} / \eta / \mathrm{ns} \mathrm{cP}^{-1}$ & $E^{*} / \mathrm{kcal} \mathrm{mol}^{-1}$ \\
PI & Denzene & 0.27 & 1.1 \\
& $o-X y r e n e$ & 1.4 & 1.3 \\
PS & Benzene & 1.1 & 1.2 \\
& Toluene & 3.1 & 1.7 \\
& Ethylbenzene & 3.2 & 1.4 \\
\multirow{2}{*}{-PMMA } & Benzene & 3.2 & 1.3 \\
& Chloroform & 4.5 & 2.7 \\
$\mathrm{PVCz}$ & THF & 3.5 & 2.4 \\
& & & 4.5 \\
\hline
\end{tabular}

for each polymer, it should be noted that the PVCz was labeled with the anthracene group through $\mathrm{C}-\mathrm{S}$ bonds, i.e., $\sim \mathrm{CHX}-\mathrm{S}-\mathrm{An}-\mathrm{S}-\mathrm{CHX} \sim$, where $\mathrm{X}$ and $\mathrm{An}$ indicate a substituent and anthracene, respectively, whereas the anthracene moieties for the other polymers were labeled with anthracene through methylene bonds; $\sim \mathrm{CHX}-\mathrm{CH}_{2}$ $-\mathrm{An}-\mathrm{CH}_{2}-\mathrm{CHX} \sim$. Shimanouchi's group studied the rotational isomerism of sulfides by infrared and Raman spectroscopy. ${ }^{47,48}$ They found that the gauche conformation for a $\mathrm{C}^{-} \mathrm{S}$ bond is as stable as the trans state, and that the bond rotation is more likely to occur around a $\mathrm{CH}_{2}-\mathrm{S}$ bond than that around a $\mathrm{CH}_{2}-\mathrm{CH}_{2}$ bond. In the previous study, we reported that the relaxation time is influenced by the chemical structure around the anthracene probe. ${ }^{49}$ Hence, the reduced relaxation time for $\mathrm{PVCz}$ evaluated in this study was probably underestimated due to the structure around the anthracene probe. The value of $T_{\mathrm{m}} / \eta$ for $\mathrm{PVCz}$ was larger than that for PS and smaller than that for $s$-PMMA. However, considering the difference in the chemical structure around the anthracene probe, it is reasonable to say that the chain mobility of PVCz is similar to that of $s$-PMMA at least. Therefore, it can be said that the relaxation time for the $\mathrm{PVCz}$ chain is fairly larger than those for the other polymers. The activation energy for $\mathrm{PVCz}$ is also larger than those for the others. These results indicate that the $\mathrm{PVCz}$ chain is less mobile and dynamically stiffer compared to other polymers. This is attributed to the steric hindrance of the bulky carbazolyl side chain. Consequently, the rotational potential barrier for the conformational transition is high and the rotational motion is suppressed.

The molecular weight effect on the relaxation time for POE and PS in good solvents was examined in detail. ${ }^{23,25}$ Now the molecular weight dependence of local chain dynamics is discussed in terms of the critical number of bonds, $N_{\mathrm{c}}$, which is the number of bonds corresponding to the critical molecular weight, $M_{\mathrm{c}} . M_{\mathrm{c}}$ is the molecular weight where the reduced relaxation time reaches an asymptotic value. The relaxation time for PS in benzene reached the saturation value at $M_{\mathrm{w}} \cong 10000$, which corresponds to $N_{\mathrm{c}}=200$. Similarly, $N_{\mathrm{c}}$ for POE in a good solvent, DMF, is estimated to be 70. On the other hand, the relaxation time for $\mathrm{PVCz}$ in $\mathrm{THF}$ was constant above a molecular weight of 25000 , which corresponds to $N_{\mathrm{c}} \cong 260$. This result means that the rotational motion around each bond for the $\mathrm{PVCz}$ backbone is suppressed due to the large steric hindrance of the carbazolyl group and more $\mathrm{C}-\mathrm{C}$ bonds are required to rotate in order to localize the segmental motion of the main chain, resulting 
in the larger critical number of bonds, $N_{\mathrm{c}}$, compared to POE and PS. The recent theoretical and numerical studies reported that the coupling of bond rotation is an important process governing the main chain dynamics. $^{12,41-43}$ When a rotational motion occurs along the main chain, counter rotation around the other bonds would cooperatively occur to avoid the movement of the large part of the polymer chain.

\section{CONCLUSION}

$\mathrm{PVCz}$ labeled with anthracene at the middle of the main chain was synthesized by the living cationic polymerization followed by the coupling reaction of the living ends with 9,10-anthracenedithiol lithium salt. The local chain dynamics of $\mathrm{PVCz}$ at the chain center was examined by the fluorescence depolarization method. The molecular weight effect upon the relaxation time and the activation energy for the local segmental motion was evaluated in the range of molecular weight from 9000 to 100000. The molecular weight and solvent dependence of the reduced relaxation time indicates that the local potential energy for the conformational transition is an important factor governing the local motion of PVCz. The values of the reduced relaxation time and the activation energy for $\mathrm{PVCz}$ were large compared to those for the other polymers, POE, PI, PS, and $s$-PMMA. Such high relaxation time and activation energy result from the suppression of the rotational motion of the backbone $\mathrm{C}-\mathrm{C}$ bonds by bulky carbazolyl side chains.

Acknowledgment. H. A. acknowledges Research Fellowships of the Japan Society for the Promotion of Science for Young Scientists.

\section{REFERENCES}

1. J. M. Pearson and M. Stolka, "POLY( $N$-VINYLCARBAZOLE)", Gordon and Breach Science Publishers, New York, N.Y., 1981.

2. A. Itaya, "Polyvinylcarbazole (in Japanease)", Bunshin, Tokyo, 1990.

3. Y. Tsujii, A. Tsuchida, Y. Onogi, and M. Yamamoto, Macromolecules, 23, 4019 (1990).

4. A. Tsuchida, A. Nagata, M. Yamamoto, H. Fukui, M. Sawamoto, and T. Higashimura, Macromolecules, 28, 1285 (1995).

5. H. Sakai, A. Itaya, H. Masuhara, K. Sasaki, and S. Kawata, Polymer, 37, 31 (1996).

6. H. Miyasaka, T. Moriyama, and A. Itaya, J. Phys. Chem., 100, 12609 (1996)

7. T. Kawakami and N. Sonoda, Appl. Phys. Lett., 62, 2167 (1993).

8. S. M. Silence, C. A. Walsh, D. M. Burland, R. J. Twieg, W. E. Moerner, and M. C. J. M. Donckers, Appl. Opt., 33, 2218 (1994).

9. M. D. Rahn, D. P. West, and J. D. Shakos, J. Appl. Phys., 87, 627 (2000).

10. H. Yamakawa, "Helical Wormlike Chains in Polymer Solutions" Springer-Verlag GmbH \& Co., Berlin, 1997.

11. E. Helfand, J. Chem. Phys., 54, 4651 (1971).

12. G. J. Moro, J. Chem. Phys., 97, 5749 (1992).
13. S. Glowinkowski, D. J. Gisser, and M. D. Ediger, Macromolecules, 23, 3520 (1990).

14. W. Zhu and M. D. Ediger, Macromolecules, 28, 7549 (1995).

15. C. Baysal, B. Erman, I. Bahar, F. Laupretre, and L. Monnerie, Macromolecules, 30, 2058 (1997).

16. A. T. Bullok, G. G. Cameron, and P. M. Smith, J. Phys. Chem., 77, 1635 (1973).

17. J. Pilar, J. Labsky, A. Marek, D. E. Budil, K. A. Earle, and J. H. Freed, Macromolecules, 33, 4438 (2000).

18. K. Adachi, "Dielectric Spectroscopy of Polymeric Materials", J. P. Runt and J. J. Fitzgerald, Ed., American Chemical Society, Washington, 1997.

19. F. R. Trouw and D. L. Price, Annu. Rev. Phys. Chem., 50, 571 (1999).

20. T. Takaeda, T. Yoshizaki, and H. Yamakawa, Macromolecules, 27, 4248 (1994).

21. A. K. Rizos and K. L. Ngai, Macromolecules, 31, 6217 (1998).

22. M. D. Ediger, Annu. Rev. Phys. Chem., 42, 225 (1991).

23. J. Horinaka, S. Amano, H. Funada, S. Ito, and M. Yamamoto, Macromolecules, 31, 1197 (1998).

24. K. Ono, K. Ueda, and M. Yamamoto, Polym. J., 26, 1345 (1994).

25. J. Horinaka, H. Aoki, S. Ito, and M. Yamamoto, Polym. J., 31, 172 (1999).

26. K. Ono, Y. Okada, S. Yokotsuka, T. Sasaki, and M. Yamamoto, Macromolecules, 27, 6482 (1994).

27. J. Horinaka, K. Ono, and M. Yamamoto, Polym. J., 27, 429 (1995).

28. M. Yamamoto, J. Horinaka, H. Aoki, K. Tawa, and S. Ito, J. Soc. Rheol. Jpn., 25, 203 (1997).

29. D. A. Waldow, M. D. Ediger, Y. Yamaguchi, Y. Matsushita, and I. Noda, Macromolecules, 24, 3147 (1991).

30. S. Adams and D. B. Adolf, Macromolecules, 31, 5794 (1998).

31. A. Karali, P. Dais, and F. Heatley, Macromolecules, 33, 5524 (2000).

32. M. Sawamoto, J. Fujimori, and T. Higashimura, Macromolecules, 20, 916 (1987).

33. E. Kobayashi, J. Jiang, H. Ohta, and J. Furukawa, J. Polym. Sci., Part A: Polym. Chem., 28, 2641 (1990).

34. A. Abe, H. Kobayashi, T. Kawamura, M. Date, T. Uryu, and K. Matsuzaki, Macromolecules, 21, 3414 (1988).

35. N. Sato, S. Ito, K. Sugiura, and M. Yamamoto, J. Phys. Chem. $A, \mathbf{1 0 3}, 3402$ (1999).

36. D. V. O'Conner and D. Phillips, "Time-Correlated Single Photon Counting" Academic Press Ltd., London, 1984.

37. S. P. Velsko, D. H. Waldeck, and G. R. Fleming, J. Chem. Phys., 78, 2735 (1983).

38. N. Kuwahara, S. Higashida, M. Nakata, and M. Kaneko, J. Polym. Sci. A-2, 7, 285 (1969).

39. G. Sitaramaiah and D. Jacobs, Polymer, 11, 165 (1970).

40. D. A. Waldow, B. S. Johnson, P. D. Hyde, M. D. Ediger, T. Kitano, and K. Ito, Macromolecules, 22, 1345 (1989).

41. N. E. Moe and M. D. Ediger, Macromolecules, 29, 5484 (1996).

42. M. M. Fuson and M. D. Ediger, Macromolecules, 30, 5704 (1997).

43. C. Baysal, A. R. Atilgen, B. Erman, and I. Bahar, Macromolecules, 29, 2510 (1996).

44. F. Abe, Y. Einaga, T. Yoshizaki, and H. Yamakawa, Macromolecules, 26, 1884 (1993).

45. F. Abe, K. Horita, Y. Einaga, and H. Yamakawa, Macromolecules, 27, 725 (1994).

46. H. A. Kramers, Physica, 7, 284 (1940).

47. M. Sakakibara, H. Matsuura, I. Harada, and T. Shimanouchi, Bull. Chem. Soc. Jpn., 50, 111 (1977).

48. M. Ohta, Y. Ogawa, H. Matsuura, I. Harada, and T. Shimanouchi, Bull. Chem. Soc. Jpn., 50, 380 (1977).

49. J. Horinaka, S. Ito, M. Yamamoto, Y. Tsujii, and T. Matsuda, Macromolecules, 32, 2270 (1999). 